\title{
The stem cell niche: tissue physiology at a single cell level \\ Jonathan Hoggatt ${ }^{1,2,3}$ and David T. Scadden ${ }^{1,2,3}$
}

${ }^{1}$ Center for Regenerative Medicine, Massachusetts General Hospital, Boston, Massachusetts, USA. 2Department of Stem Cells and Regenerative Biology, Harvard University, Cambridge, Massachusetts, USA. ${ }^{3}$ Harvard Stem Cell Institute, Cambridge, Massachusetts, USA.

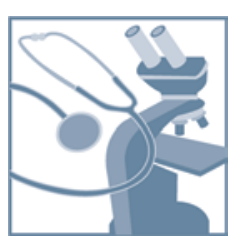

\begin{abstract}
Stem cells are the critical unit affecting tissue maintenance, regeneration, and repair, with particular relevance to the tissues with high cell turnover. Stem cell regulation accommodates the conflicting needs of prompt responsiveness to injury and long-term preservation through quiescence. They are, in essence, the fundamental unit by which a tissue handles changing physiologic needs throughout the lifetime of the organism. As such, they are the focal point of dynamic tissue function, and their governance is physiology expressed at a cellular and molecular level. Here, we discuss the multiple components representing the stem cell niche in hematopoiesis and argue for a unbiased mapping of the niche constituents under different conditions as the first step in developing systems physiology.
\end{abstract}

\section{Introduction}

The concept of a specialized microenvironment governing stem cell behavior is now commonly accepted, with cells contributing to the niche of tissues, like blood, gut, skin, and brain, increasingly being defined. While simple regulatory relationships between stem cell and niche elements are the substance of most studies following the model so well defined in invertebrates, the list of participants, in the bone marrow in particular, has become sufficiently rich that models of niche function must begin to accommodate complexity. Accomplishing this requires both a means of delineating contributions of individual parts and a clear sense of what the functional outcomes of greatest interest are. In its earliest and simplest formulation, the niche hypothesis described a heterologous cell interaction, fostering the preservation of the stem cell state. However, the functions of the niche have taken on new dimension, in keeping with an evolving sense of how stem cells behave, in parallel with an increasingly diverse array of participating elements of the microenvironment that regulates them. In the tissues with high turnover (gut, airway, skin, and blood) that provide critical defense from the outside world, there is a highly ordered production of massive numbers of cells, a process fraught with danger to a long-lived animal like the human. To put the production demands of the system in perspective, the number of cells produced daily to simply maintain the hematopoietic system alone exceeds the estimated number of stars in the Milky Way (1). The tens of millions of mitoses required per minute pose the inevitable consequence of mutation and potential exhaustion. The adverse consequences of mutation are mitigated by the organization of subpopulations within the tissues. That organizational schema follows the general rule of self-renewal being restricted to a stem cell pool of limited size and proliferative activity, thereby reducing the likelihood of accumulated genetic injury in any given cell: mutations in non-self-renewing progenitors would be of modest consequence if the cells inexorably progress toward death. However, it is now clear that in at least some high turnover systems, like the gut (2-4) and blood (5-8), stem cells are not uniformly qui-

Conflict of interest: The authors have declared that no conflict of interest exists. Citation for this article: J Clin Invest. 2012;122(9):3029-3034. doi:10.1172/JCI60238. escent. This variable behavior of stem cells raises the notion that niche components provide a means by which the stem cell state is preserved and also participate in governing the relative proliferative activity of stem cells.

Through modification of specific molecules in either a broad range of cells from the microenvironment or a selected subset, it is now clear that the microenvironment serves to integrate proliferative and differentiation events in hematopoiesis. For example, perturbation of RAR $\gamma$ signaling or $\mathrm{Rb}$ and $\mathrm{p} 53$ expression in the microenvironment results in myeloproliferative phenotypes with remote tissue infiltration $(9,10)$. Perhaps most strikingly, altering genes for the miRNA-processing enzymes, DICER, DROSHA, or DGCR8, or the ribosomal complex gene, $S B D S$, in osteolineage cells created a complex hematopoietic phenotype with multiple defects at the stem cell, progenitor, and mature cell level, creating a myelodysplasia-like state (11). The progression of stem cells to progenitors and mature cells was disordered sufficiently to compromise the number and even morphologic appearance of the cells. These hematopoietic effects seem to be dependent upon a particular subset of osteolineage cells, specifically those expressing OSTERIX, a protein preferentially expressed in immature cells. No hematopoietic phenotype was seen when DICER1 was deleted in mature osteoblasts. The changes that were seen in hematopoietic function were entirely microenvironment dependent, as transplants of mutant cells into unmodified hosts reversed the myelodysplasia, and transplant of wild type cells into the modified host induced the phenotype (11). These data indicate that specific subsets of cells in the environment govern how the stem cell proceeds in its generation of its descendent cells.

Strikingly, in the DICER deletion model, a small number of animals developed an acute myeloid leukemia that involved multiple new genetic lesions, as shown by comparative genomic hybridization analysis (11). These data strongly support the notion that cells of the microenvironment are imposing constraints on the hematopoietic cells that when altered select for hematopoietic cells that may have acquired different function due to de novo mutations. The niche may indeed be imposing "fitness" determinants on hematopoietic stem and progenitor cells (HSPCs), and when these fitness parameters change, abnormal cells may have 
a competitive advantage. Another study further supporting that concept demonstrated that HSCs expressing the oncoprotein AML1-ETO were preferentially supported when transplanted into aged compared with young animals (12). Niche characteristics may therefore be providing selective pressures enforcing particular stem cell characteristics by granting them competitive privilege.

The niche exerting fitness pressures on the stem cell is particularly intriguing in hematopoiesis because of the known circulation of those stem cells in the adult. The question of why stem cells continue to circulate in the adult mammal well after the establishment of hematopoiesis in the bone marrow has long been perplexing. Part of the basis for this has been thought to involve response to ongoing variations in cytokine levels, such as G-CSF, which affect expression of tethering molecules in the bone marrow (13). It is also increasingly recognized that structural elements within the niche such as osteolineage cells are undergoing substantial turnover under homeostatic conditions (14). Therefore, the HSC may essentially lose its niche periodically, as some of the cells that comprise it terminate. Neither of these scenarios fully explains why cells would be in the circulation and not just in the interstitial, extracellular space within the marrow, but both suggest dynamism of place that may implicitly impose a requirement for stem cells to effectively find themselves a new niche, with attendant demands on localization and engraftment functions. These requirements impose a de facto fitness challenge on the stem cells that may also offer some selective advantage for the organism.

Therefore, the niche should be regarded as a dynamic participant in the health of the tissue and the organism. Because the stem cell is at the root of tissue sustenance, it is somewhat expected that it will be multiply determined. It is also not surprising that the stem cell will be regulated by processes that can be modulated, are likely redundant, and are perhaps reciprocal in function to achieve outcomes reflective of complex organismal need. Studying complex tissue components that in sum affect stem cell function is a daunting challenge and is only minimally served by a reductionist approach. Yet, thus far, it is by reductionist means that we have learned anything at all about the niche. A summary of the recent findings in niche participants follows, along with a perspective on how we might take the necessary next step in untangling the weave of the niche.

\section{Ultrastructure of the stem cell niche}

HSPCs are not randomly located in the bone marrow, but preferentially reside in trabecular bone and are relatively infrequent in regions such as the diaphyses of long bones (15). From studies of cell position relative to progenitor function conducted in the 1970 s, it was noted that the endosteal surfaces of bone are a preferential location for primitive hematopoietic cells $(16,17)$. With more precise in vivo microscopy, it is evident that transplanted cells take a position determined by their cell state. That is, HSCs localize to endosteal surfaces, while more mature hematopoietic progenitor cells localize further away (18-21), and HSCs tightly associated with the endosteum have been shown to have greater transplantation activity than those in the central marrow cavity (22). HSCs from aged donors were also shown to localize to sites further away from the endosteum than those from younger donors (18), suggesting that age influences interactions within the niche. Therefore, there is a coherent logic to marrow macroarchitecture, in which hematopoietic cell state and cell position correlate and yet can be modulated.
Given these earlier localization studies identifying the endosteum as a localization point for HSCs, osteolineage cells lining the endosteum were recognized as potential HSC supporters via in vitro studies by Taichman and colleagues $(23,24)$ and later through a series of in vivo studies (25-28). It should be noted that we have chosen to use the term "osteolineage cells" to describe these populations of HSC supportive cells, while many of these previous publications referred to these cells as "osteoblasts" due to cell morphology, endosteal localization, and various phenotypic markers. However, it has recently become clear that osteolineage cells exist on a spectrum of differentiation, similar to hematopoietic cells, and it is likely that these earlier studies encompassed an earlier component cell. It is the earlier osteolineage cell that appears to be involved in HSC regulation (11).

The populations of cells comprising the marrow in trabecular bone are highly complex, as it is a rich network of vessels, nerves, bone-remodeling elements, and subpopulations of hematopoietic cells comprising a region whose three-dimensional architecture crudely resembles that of sponge (Figure 1). The positioning of HSPCs in relation to these elements is variable. After transplantation, HSPCs were noted in CXCL12-rich vascular zones, in which they were positioned longer term and appeared to proliferate over time (29). By histology a large fraction of HSCs were seen localized to sinusoidal endothelium, with only a portion of HSCs associated with the endosteum (30). The presence of HSPCs next to vessels could simply reflect the cells in transit, a phenomenon that is known to occur with high frequency (31). However, the proportion of HSPCs in the perivascular position is large, and there is now ample evidence of perivascular mesenchymal cells playing a role in regulating HSPC function (32-34).

HSPCs residing in regions relatively equidistant from both endosteal lining cells and perivascular/sinusoidal components may be subject to a set of different conditions than those at a greater distance from the endosteum. For example, the endosteal surface bone remodeling activity is thought to generate high ionic calcium levels, and the calcium-sensing receptor is known to be expressed on HSPCs and affect their function (35). Further, the types of mesenchymal cells on the endosteal surface are different than those in the marrow interstitium, in that they include bone matrix-producing cells that elaborate distinct secreted and cell surface proteins such as agrin (36) and osteopontin (with higher protein levels at the endosteum; ref. 37), both defined HSPC regulators. Finally, the presence of the bone matrix itself also creates a distinct biophysical surface that has yet to be fully explored, but is likely to provide a unique set of interactions for HSPCs or the molecules that regulate them.

Those perivascular HSPCs at a distance from the endosteum may not just be exposed to fewer of the endosteal-specific components, but may also be engaging a distinct set of cells and their signals. The mesenchymal populations in the marrow are highly heterogeneous. Some, like osteolineage-committed cells, have a known position on the endosteum. Genetic manipulation of these cells has been shown to alter HSPC number, proliferative activity, and sensitivity to the mobilizing signal of $\operatorname{G-CSF}(8,11,25,38)$. A number of primitive mesenchymal cells with multilineage or osteolineage potential have been identified in lineage-tracing studies and these have been characterized as nestin-positive (32) or Mx-1-expressing cells (14). Others are of unclear differentiation potential but express leptin receptor and high levels of kit ligand (39). Deletion of kit ligand in the leptin receptor-positive population markedly reduced bone marrow HSCs in a manner that was 


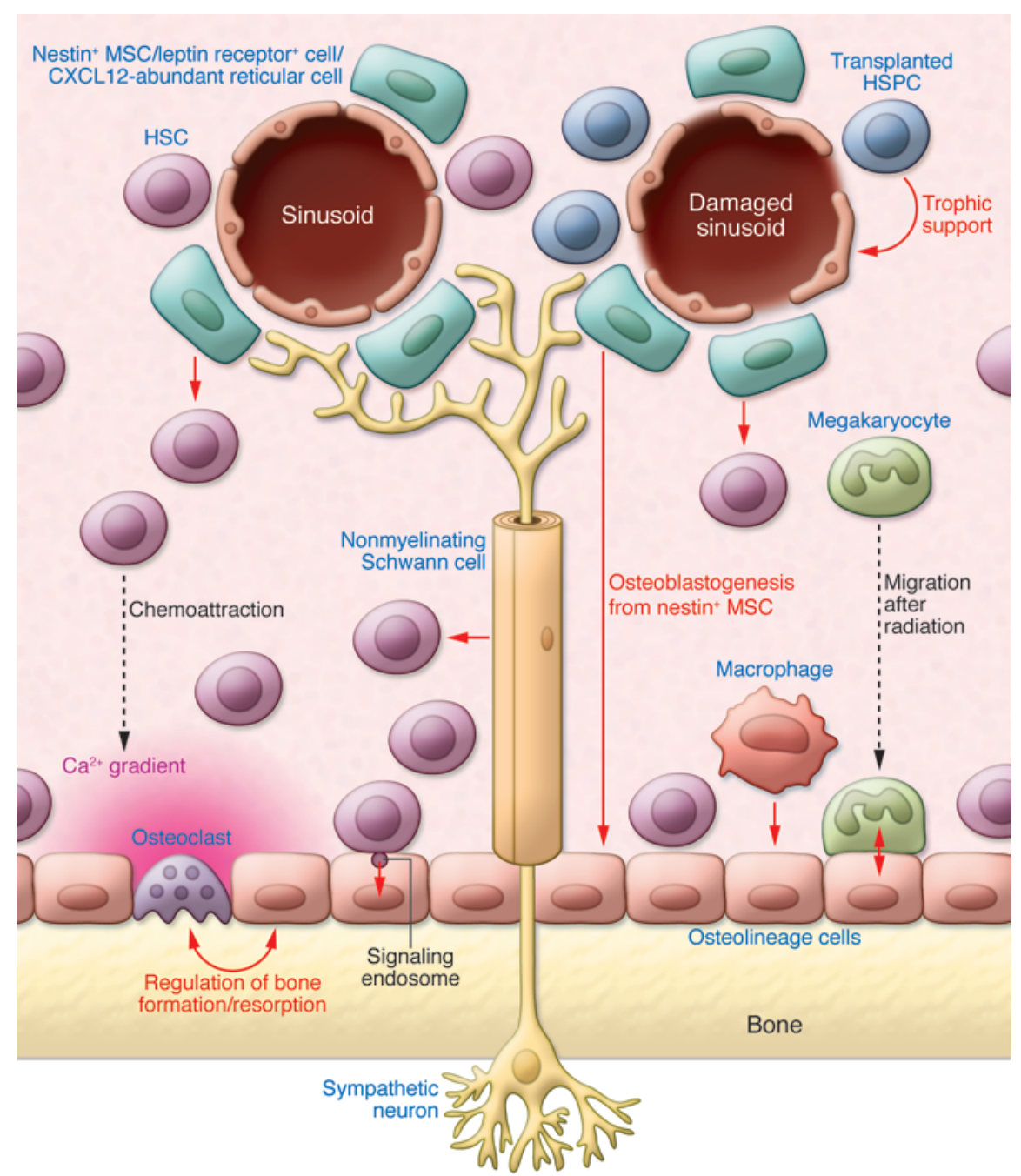

\section{Figure 1}

Schematic representation of the HSC niche. HSCs reside in bone marrow niches, closely associated with osteolineage cells, the highly innervated nestin+ mesenchymal stem cells (MSCs), perivascular CXCL12-abundant reticular cells, or sinusoidal lining leptin receptor $^{+}$cells, with overlapping regions and differing oxygen tensions. Resident macrophages provide a positive supporting factor maintaining osteolineage and mesenchymal stem cell activity, and megakaryocytes support osteolineage cells after injury through cell-to-cell contact. Similarly, HSCs making cell-to-cell contact can create signaling endosomes, enhancing osteolineage hematopoietic support, and transplanted HSCs facilitate sinusoidal endothelial repair following injury. Osteoclasts, in conjunction with osteoblasts, maintain bone formation and resorption, creating a soluble calcium gradient, which is a chemoattractant for HSCs. SNS signals regulate bone marrow niche components, and nonmyelinating Schwann cells ensheathing those neurons can directly regulate HSCs through TGF- $\beta$ production. not seen when the same gene deletion was performed in nestinexpressing or osteolineage cells (39).

There are also mesenchymal populations that are not necessarily perivascular that influence hematopoiesis. A CXCL12 ${ }^{+}$ mesenchymal cell of ambiguous function, termed the CXCL12abundant reticular cell, was found to be the predominate CXCL12expressing cell within the bone marrow and was found scattered throughout the intertrabecular space of the bone marrow cavity and adjacent to sinusoidal endothelial cells (34). Adipocytes also can affect HSPCs, by negatively regulating their number (40). These data all point to distinctive sub-environments in the marrow that may contribute variegated signals to HSPCs.

It is fair to conclude then that hematopoietic cells have differentiation stage-specific, or age-specific, positions, and their positioning exposes them to differing conditions. A popular hypothesis holds that the positioning corresponds to the increasingly recognized heterogeneity of the HSPC pool in terms of functions like cell cycling or responsiveness to mobilization signals, though that is yet to be experimentally defined.

\section{Niche connectivity}

Physiology is the study of the functional response to differing needs. If the niche is to be considered a quintessential expression of tissue physiology, it follows that it should have a high degree of connectedness to the state of the tissue and the organism. Perhaps the greatest manifestation of that connectedness is the link to the ultimate integrator of organism level information, the central nervous system. In a series of extraordinarily insightful studies, Paul Frenette's laboratory defined how the sympathetic nervous system (SNS) participates in HSPC regulation largely through $\beta$-adrenergic signals altering the function of mesenchymal cells, changing their expression of known mediators of HSPC localization, such as the chemokine, CXCL12, and its receptor, CXCR4 $(32,41,42)$. Modulation of sympathetic tone occurs with central circadian oscillators, and the same group showed how rhythmic SNS activity results in cyclic changes in HSPC mobilization into the peripheral blood $(43,44)$. These data conclusively show that the hematopoietic niche is at the command of the highest order of organismal physiology, the brain.

Our understanding of how other nervous system components participate in the niche took an interesting turn when Yamazaki et al. described a population of nonmyelinating Schwann cells as HSPC regulators (45). These cells ensheathe sympathetic nerves in the bone marrow and were shown to be a primary producer of TGF- $\beta$, which Yamazaki and others have demonstrated is a quiescent signal for HSCs $(46,47)$. When the bone was denervated, TGF- $\beta$ signaling was reduced, leading to a reduction in the HSC 
pool (45). Thus, nervous system participation extends beyond events at the synaptic nerve terminal. The recent demonstration that hematopoietic cells in Drosophila larva rely on peripheral nervous system signaling (48) argues that connectivity between nervous and hematopoietic systems is highly conserved (49).

The vascular tree may also be regarded as a source of integration. We had previously shown, in collaboration with the laboratory of Charles Lin, that vascular subdomains exist in the bone marrow with abundant CXCL12 and E-selectin expression and that HSPCs preferentially localized to these sites, suggesting niche function (29). It has recently been shown that regeneration of sinusoidal endothelial cells is essential for hematopoietic reconstitution following myelosuppression (50) and bone marrow endothelial cells support the growth and expansion of HSCs ex vivo through the expression of "angiocrine" factors (51). In addition, the deletion of kit ligand in tie2-expressing endothelial cells results in a loss of HSCs from the bone marrow (39). Therefore, both a subset of endothelial cells and the mesenchymal cells that are adjacent to them participate in the niche. Their connection to circulating factors reflecting the state of the tissue and organism is a likely means by which host physiology broadly conceived can influence the HSC-driven hematopoietic response.

One such simple metabolic parameter in which the vasculature directly participates is that of oxygen tension. While oxygenation levels in the bone marrow are controversial, it is clear that oxygenresponsive genes alter niche function. HIF- $1 \alpha$-deficient mice have reduced expression of CRIPTO on endosteal osteolineage cells and a reduction of one of CRIPTO's cognate receptors, GRP78, on HSCs (52). The CRIPTO/GRP78 signaling axis was shown to be an important regulator of HSC quiescence downstream of HIF- $1 \alpha$ signaling, and it was postulated that GRP78 could be used as a marker to distinguish between quiescent and active HSCs. In mice in which the HIF- $1 \alpha$-responsive element on the Vegfa promoter was mutated, thereby preventing upregulated expression in hypoxic conditions, HSCs were found to have defects in their function (53), supporting a role of hypoxia-induced gene expression in the niche for maintenance of HSCs. These follow other studies in which HIF- $1 \alpha$ has been shown to be necessary for HSPC cell cycle quiescence and resistance to stress (54). HIF-1 $\alpha$ was also shown to increase production of CXCL12 (55) and CXCR4 (56) and prevent reactive oxygen species-mediated HSPC damage (57). It is somewhat obvious that oxygen levels will have multiple means of altering HSPC function, including affecting niche function, but we are just beginning to gain granularity on how and where these alterations occur. It will take new technologies to accurately measure oxygen tension within the bone marrow microenvironment at high resolution to determine how oxygen levels themselves change and modify specific cells within the architecture of the marrow under different physiologic states.

\section{Bidirectional niche biology}

Connections of systemic inputs to HSPC function are generally the focus of niche studies, but recent evidence intriguingly posits that information may flow in reverse. Human CD $34^{+} \mathrm{HSPCs}$ cocultured with osteolineage cells were observed to exchange a portion of their membrane with the osteolineage cells, creating a signaling endosome (58). This caused osteolineage cells to downregulate SMAD signaling and increase production of CXCL12. While these data were derived exclusively from in vitro studies, they raise the interesting possibility that hematopoietic cells may instruct osteolineage cells to create a more habitable environment, allowing them to directly affect their own microenvironment. This may suggest a concept that has long been proposed based on clinical observations under conditions in which the marrow space is infiltrated or demands of hematopoiesis are extreme: that the HSC can instruct surrounding cell neighbors to form a supportive microenvironmental niche.

Mature hematopoietic cells certainly have the ability to alter niche function, and three recent reports indicate a new role for macrophages in that capacity (59-61). Macrophages characterized as $\mathrm{F} 4 / 80^{+} \mathrm{Ly}-6 \mathrm{G}^{+} \mathrm{CD} 11 \mathrm{~b}^{+}(61)$ or $\mathrm{Gr}-1^{\text {negative }} \mathrm{F} 4 / 80^{+} \mathrm{CD} 115^{\text {mid }}$ $\mathrm{CD}_{169}{ }^{+}(59)$ line the endosteal surfaces of bone and are reduced in number by G-CSF treatment. Normally, these macrophages produce soluble factors promoting osteolineage function and subsequent production of supportive hematopoietic niche molecules like SDF-1 $\alpha$ (59-61). When macrophages are reduced in bone marrow with G-CSF treatment, this positive support on osteolineage cells is lost, resulting in an attenuation of the hematopoietic niche and HSPC mobilization to the periphery. A distinct monocyte lineage, osteoclasts, has also been previously reported to increase HSPC mobilization when stimulated by RANK ligand, due to cathepsin $\mathrm{K}$ degradation of tethering molecules $(62,63)$. Recent data indicating that bisphosphonate inhibition of osteoclast function did not perturb HSPC mobilization and that osteoclast proliferation in response to G-CSF may antedate mobilization suggest that the role of osteoclasts may be more nuanced $(61,64,65)$.

Megakaryocytes may affect niche components as well. For example, after irradiation, megakaryocytes were shown to migrate toward the endosteal surface and were implicated in an expansion in osteolineage cells (66). Mice deficient in GATA-1 and NF-E2 transcription factor expression had significantly increased megakaryocyte numbers and a 6-fold expansion of osteolineage cells (67) dependent on direct cell-cell contact between megakaryocytes and osteolineage cells $(68,69)$.

While some indications of hematopoietic cell modification of niche function have been defined, this remains a generally understudied topic and is of particular interest when considering how feedback loops between HSPCs and their niche may tune the relative abundance of each during conditions such as growth, transplantation, or leukemia.

\section{Systems niche biology}

As new roles for the HSPC microenvironment and new components that comprise it are defined, it is apparent that the ecological concept first applied to it by Schofield (70), a niche, was prescient. There is an interweaving of niche, HSPC, and organismal activities that can be seen as connected biomes that are in dynamic equipoise, with any change in one altering the activity of the other. While reductionist experimental approaches have lead to considerable advances in our understanding of the niche, it is clear that the interconnectedness and complexity of the system requires a broader view. So how to proceed?

Complexity of interactions is far from unique to the niche and is a common problem facing biologists, whether they are focused on the molecular, cellular, or organismal levels. By systematically gathering unbiased and comprehensive data sets under varying conditions, "omics" approaches are offering insights into internal logic and nodes of control. While such an approach is extremely challenging when applied to tissue, the current process of unveiling single elements sequentially is increasingly revealing its limi- 
tations. The parable of blind men describing the elephant in entirely different terms depending on which part of the animal they happened to encounter is a fitting description of our current process. Notably, when authoring a piece on Schofield's original conception of the niche some years ago, one of us (D.T. Scadden) requested a picture of Schofield from his colleague, Brian Lord. Within the background of the photograph in Schofield's office is a drawing of blind men examining an elephant. Well before any experimental definition of the concept he provided was acquired, Schofield's awareness of how standard reductionist approaches would restrict our vision was strikingly sage.

If reductionism is reaching its asymptote of utility, the alternative is to gather data in an unbiased manner, systematically. While many approaches may apply, what is ultimately required is to accomplish understanding of geographic relationships on a microscopic level within intact, physiologic niches. This would involve quantifying cell types, numbers of those types, levels of gene expression of those types of cells, and correlations of how the products of expression of those genes in those cells alter the outcome of blood cell production and preservation under varying types of physiologic stress. That is an enormous undertaking, but it is one that is becoming possible to envision. There are emerging technologies combining microscopy with molecular assessment that can be either highly multiplexed or unbiased. Those approaches may enable molecular signatures of cells with physical proximity to HSPCs to be defined without prior knowledge of the cell type. Those signatures can be used to bin cell populations, with subsequent quantification of cells in those bins under specific physiologic conditions. The perturbations of particular molecules within the bin populations corresponding to changes in HSPC number, function, and outcome in the blood could be enumerated, and correlations could be computationally established. These first steps would only begin to highlight what would need to be subsequently accomplished through more traditional means of candidate validation. However, they would begin to map in a more comprehensive manner the parts and interactions of the parts of the elephant. This would be a first step at understanding the tissue systems physiology that the HSC and its niche represent. It may be the only way to make sense of the complex interconnectedness of the niche and to avoid niche biology being a collection of halfblind observations. It may also inform strategies for altering the niche to achieve clinically important effects.

\section{Acknowledgments}

J. Hoggatt is supported by NIH training grant HL087735 and is a consultant for Fate Therapeutics. D.T. Scadden is supported by the NIH (HL44851, HL100402, HL97794, HL108655, and CA1631910), the Ellison Medical Foundation, and the Peabody Foundation and is a consultant for Fate Therapeutics, Hospira, GSK, and Bone Therapeutics.

Address correspondence to: David Scadden, Massachusetts General Hospital, Center for Regenerative Medicine, 185 Cambridge Street, CPZN-4265A, Boston, Massachusetts 02114, USA. Phone: 617.726.5615; Fax: 617.724.2662;E-mail: david_scadden@ harvard.edu.
1. Simon S. The Heart: Our Circulatory System. New York, New York, USA: Harper Collins; 2006.

2. Yan KS, et al. The intestinal stem cell markers Bmi1 and Lgr5 identify two functionally distinct populations. Proc Natl Acad Sci U S A. 2012; 109(2):466-471.

3. Carlone DL, Breault DT. Slowly cycling versus rapidly cycling intestinal stem cells: distinct roles or redundancy. Cell Cycle. 2011;10(5):723-724.

4. Simons BD, Clevers H. Stem cell self-renewal in intestinal crypt. Exp Cell Res. 2011;317(19):2719-2724.

5. Glauche I, Moore K, Thielecke L, Horn K, Loeffler M, Roeder I. Stem cell proliferation and quiescence-two sides of the same coin. PLoS Comput Biol. 2009;5(7):e1000447.

6. van der Wath RC, Wilson A, Laurenti E, Trumpp A, Lio P. Estimating dormant and active hematopoietic stem cell kinetics through extensive modeling of bromodeoxyuridine label-retaining cell dynamics. PLoS One. 2009;4(9):e6972.

7. Foudi A, et al. Analysis of histone 2B-GFP retention reveals slowly cycling hematopoietic stem cells. Nat Biotechnol. 2009;27(1):84-90.

8. Wilson A, et al. Hematopoietic stem cells reversibly switch from dormancy to self-renewal during homeostasis and repair. Cell. 2008;135(6):1118-1129.

9. Walkley CR, Shea JM, Sims NA, Purton LE, Orkin $\mathrm{SH}$. Rb regulates interactions between hematopoietic stem cells and their bone marrow microenvironment. Cell. 2007;129(6):1081-1095

10. Walkley CR, et al. A microenvironment-induced myeloproliferative syndrome caused by retinoic acid receptor gamma deficiency. Cell. 2007; 129(6):1097-1110.

11. Raaijmakers $\mathrm{MH}$, et al. Bone progenitor dysfunction induces myelodysplasia and secondary leukaemia. Nature. 2010;464(7290):852-857.

12. Vas V, Wandhoff C, Dorr K, Niebel A, Geiger H. Contribution of an aged microenvironment to aging-associated myeloproliferative disease. PLoS One. 2012;7(2): 31523.
13. Abkowitz JL, Robinson AE, Kale S, Long MW, Chen J. Mobilization of hematopoietic stem cells during homeostasis and after cytokine exposure. Blood. 2003;102(4):1249-1253.

14. Park D, et al. Endogenous bone marrow MSCs are dynamic, fate-restricted participants in bone maintenance and regeneration. Cell Stem Cell. 2012; 10(3):259-272.

15. Ellis SL, et al. The relationship between bone, hemopoietic stem cells, and vasculature. Blood. 2011; 118(6):1516-1524.

16. Gong JK. Endosteal marrow: a rich source of hematopoietic stem cells. Science. 1978; 199(4336):1443-1445.

17. Lord BI, Testa NG, Hendry JH. The relative spatial distributions of CFUs and CFUc in the normal mouse femur. Blood. 1975;46(1):65-72.

18. Kohler A, et al. Altered cellular dynamics and endosteal location of aged early hematopoietic progenitor cells revealed by time-lapse intravital imaging in long bones. Blood. 2009;114(2):290-298.

19. Lo CC, et al. Live-animal tracking of individual haematopoietic stem/progenitor cells in their niche. Nature. 2009;457(7225):92-96.

20. Nilsson SK, Johnston HM, Coverdale JA. Spatial localization of transplanted hemopoietic stem cells: inferences for the localization of stem cell niches. Blood. 2001;97(8):2293-2299.

21. Xie Y, et al. Detection of functional haematopoietic stem cell niche using real-time imaging. Nature. 2009; 457(7225):97-101.

22. Haylock DN, et al. Hemopoietic stem cells with higher hemopoietic potential reside at the bone marrow endosteum. Stem Cells. 2007;25(4):1062-1069.

23. Taichman RS, Emerson SG. Human osteoblasts support hematopoiesis through the production of granulocyte colony-stimulating factor. J Exp Med. 1994;179(5):1677-1682.

24. Taichman RS, Reilly MJ, Emerson SG. Human osteoblasts support human hematopoietic progenitor cells in vitro bone marrow cultures. Blood.
1996;87(2):518-524.

25. Calvi LM, et al. Osteoblastic cells regulate the haematopoietic stem cell niche. Nature. 2003; 425(6960):841-846

26. Zhang J, et al. Identification of the haematopoietic stem cell niche and control of the niche size. Nature. 2003;425(6960):836-841.

27. Visnjic D, Kalajzic Z, Rowe DW, Katavic V, Lorenzo J, Aguila HL. Hematopoiesis is severely altered in mice with an induced osteoblast deficiency. Blood. 2004;103(9):3258-3264

28. Arai F, et al. Tie2/angiopoietin-1 signaling regulates hematopoietic stem cell quiescence in the bone marrow niche. Cell. 2004;118(2):149-161.

29. Sipkins DA, et al. In vivo imaging of specialized bone marrow endothelial microdomains for tumour engraftment. Nature. 2005;435(7044):969-973.

30. Kiel MJ, Yilmaz OH, Iwashita T, Yilmaz OH, Terhorst C, Morrison SJ. SLAM family receptors distinguish hematopoietic stem and progenitor cells and reveal endothelial niches for stem cells. Cell. 2005; 121(7):1109-1121.

31. Wright DE, Wagers AJ, Gulati AP, Johnson FL, Weissman IL. Physiological migration of hematopoietic stem and progenitor cells. Science. 2001; 294(5548):1933-1936.

32. Mendez-Ferrer S, et al. Mesenchymal and haematopoietic stem cells form a unique bone marrow niche. Nature. 2010;466(7308):829-834.

33. Sacchetti B, et al. Self-renewing osteoprogenitors in bone marrow sinusoids can organize a hematopoietic microenvironment. Cell. 2007;131(2):324-336.

34. Sugiyama T, Kohara H, Noda M, Nagasawa T. Maintenance of the hematopoietic stem cell pool by CXCL12-CXCR4 chemokine signaling in bone marrow stromal cell niches. Immunity. 2006; 25(6):977-988.

35. Adams GB, et al. Stem cell engraftment at the endosteal niche is specified by the calcium-sensing receptor. Nature. 2006;439(7076):599-603.

36. Mazzon C, et al. The critical role of agrin in 
the hematopoietic stem cell niche. Blood. 2011; 118(10):2733-2742.

37. Stier S, et al. Osteopontin is a hematopoietic stem cell niche component that negatively regulates stem cell pool size. J Exp Med. 2005;201(11):1781-1791.

38. Ferraro F, et al. Diabetes impairs hematopoietic stem cell mobilization by altering niche function. Sci Transl Med. 2011;3(104):104ra101.

39. Dang L, Saunders TL, Enikolopov G, Morrison SJ. Endothelial and perivacular cells maintain haematopoietic stem cells. Nature. 2012;481(7382):457-462.

40. Naveiras O, Nardi V, Wenzel PL, Hauschka PV, Fahey F, Daley GQ. Bone-marrow adipocytes as negative regulators of the haematopoietic microenvironment. Nature. 2009;460(7252):259-263.

41. Katayama Y, et al. Signals from the sympathetic nervous system regulate hematopoietic stem cell egress from bone marrow. Cell. 2006;124(2):407-421.

42. Méndez-Ferrer S, Battista M, Frenette PS. Cooperation of beta(2)- and beta(3)-adrenergic receptors in hematopoietic progenitor cell mobilization. Ann NY Acad Sci. 2010;1192:139-144.

43. Lucas D, Battista M, Shi PA, Isola L, Frenette PS. Mobilized hematopoietic stem cell yield depends on species-specific circadian timing. Cell Stem Cell. 2008;3(4):364-366.

44. Mendez-Ferrer S, Lucas D, Battista M, Frenette PS. Haematopoietic stem cell release is regulated by circadian oscillations. Nature. 2008;452(7186):442-447.

45. Yamazaki S, et al. Nonmyelinating schwann cells maintain hematopoietic stem cell hibernation in the bone marrow niche. Cell. 2011;147(5):1146-1158.

46. Larsson J, Karlsson S. The role of Smad signaling in hematopoiesis. Oncogene. 2005;24(37):5676-5692.

47. Yamazaki S, Iwama A, Takayanagi S, Eto K, Ema H, Nakauchi H. TGF-beta as a candidate bone marrow niche signal to induce hematopoietic stem cell hibernation. Blood. 2009;113(6):1250-1256.

48. Makhijani K, Alexander B, Tanaka T, Rulifson E, Bruckner $K$. The peripheral nervous system supports blood cell homing and survival in the Drosophila larva. Development. 2011;138(24):5379-5391.
49. Bruckner K. Blood cells need glia, too: a new role for the nervous system in the bone marrow niche. Cell Stem Cell. 2011;9(6):493-495.

50. Hooper AT, et al. Engraftment and reconstitution of hematopoiesis is dependent on VEGFR2-mediated regeneration of sinusoidal endothelial cells. Cell Stem Cell. 2009;4(3):263-274.

51. Kobayashi H, et al. Angiocrine factors from Aktactivated endothelial cells balance self-renewal and differentiation of haematopoietic stem cells. Nat Cell Biol. 2010;12(11):1046-1056.

52. Miharada K, et al. Cripto regulates hematopoietic stem cells as a hypoxic-niche-related factor through cell surface receptor GRP78. Cell Stem Cell. 2011; 9(4):330-344

53. Rehn M, et al. Hypoxic induction of vascular endothelial growth factor regulates murine hematopoietic stem cell function in the low-oxygenic niche. Blood. 2011;118(6):1534-1543.

54. Takubo K, et al. Regulation of the HIF-1alpha level is essential for hematopoietic stem cells. Cell Stem Cell. 2010;7(3):391-402.

55. Ceradini DJ, et al. Progenitor cell trafficking is regulated by hypoxic gradients through HIF-1 induction of SDF-1. Nat Med. 2004;10(8):858-864.

56. Staller P, Sulitkova J, Lisztwan J, Moch H, Oakeley EJ, Krek W. Chemokine receptor CXCR4 downregulated by von Hippel-Lindau tumour suppressor pVHL. Nature. 2003;425(6955):307-311.

57. Kirito K, Hu Y, Komatsu N. HIF-1 prevents the overproduction of mitochondrial ROS after cytokine stimulation through induction of PDK-1. Cell Cycle. 2009;8(17):2844-2849.

58. Gillette JM, Larochelle A, Dunbar CE, LippincottSchwartz J. Intercellular transfer to signalling endosomes regulates an ex vivo bone marrow niche. Nat Cell Biol. 2009;11(3):303-311.

59. Chow A, et al. Bone marrow CD169+ macrophages promote the retention of hematopoietic stem and progenitor cells in the mesenchymal stem cell niche. J Exp Med. 2011;208(2):261-271.

60. Christopher MJ, Rao M, Liu F, Woloszynek JR, Link
DC. Expression of the G-CSF receptor in monocytic cells is sufficient to mediate hematopoietic progenitor mobilization by G-CSF in mice. J Exp Med. 2011;208(2):251-260

61. Winkler IG, et al. Bone marrow macrophages maintain hematopoietic stem cell (HSC) niches and their depletion mobilizes HSCs. Blood. 2010; 116(23):4815-4828

62. Kollet O, et al. Osteoclasts degrade endosteal components and promote mobilization of hematopoietic progenitor cells. Nat Med. 2006;12(6):657-664.

63. Shivtiel S, et al. CD45 regulates retention, motility, and numbers of hematopoietic progenitors, and affects osteoclast remodeling of metaphyseal trabecules. J Exp Med. 2008;205(10):2381-2395.

64. Miyamoto K, et al. Osteoclasts are dispensable for hematopoietic stem cell maintenance and mobilization. J Exp Med. 2011;208(11):2175-2181.

65. Takamatsu Y, Simmons PJ, Moore RJ, Morris HA, To LB, Levesque JP. Osteoclast-mediated bone resorption is stimulated during short-term administration of granulocyte colony-stimulating factor but is not responsible for hematopoietic progenitor cell mobilization. Blood. 1998;92(9):3465-3473.

66. Dominici $M$, et al. Restoration and reversible expansion of the osteoblastic hematopoietic stem cell niche after marrow radioablation. Blood. 2009;114(11):2333-2343.

67. Kacena MA, et al. Megakaryocyte-osteoblast interaction revealed in mice deficient in transcription factors GATA-1 and NF-E2. J Bone Miner Res. 2004; 19(4):652-660.

68. Ciovacco WA, et al. The role of gap junctions in megakaryocyte-mediated osteoblast proliferation and differentiation. Bone. 2009;44(1):80-86.

69. Ciovacco WA, Cheng YH, Horowitz MC, Kacena MA. Immature and mature megakaryocytes enhance osteoblast proliferation and inhibit osteoclast formation. J Cell Biochem. 2010;109(4):774-781.

70. Schofield R. The relationship between the spleen colony-forming cell and the haemopoietic stem cell. Blood Cells. 1978;4(1-2):7-25. 